\title{
EXPONENTIALLY PREINVEX FUZZY MAPPINGS AND FUZZY EXPONENTIALLY MIXED VARIATIONAL-LIKE INEQUALITIES
}

\section{MUHAMMAD BILAL KHAN1, MUHAMMAD ASLAM NOOR1, KHALIDA INAYAT NOOR1, HASSAN ALMUSAWA ${ }^{2}$, KOTTAKKARAN SOOPPY NISAR ${ }^{3, *}$}

\author{
${ }^{1}$ Department of Mathematics, COMSATS University Islamabad, Pakistan \\ 2Department of Mathematics, College of Sciences, Jazan University, Jazan 45142, Saudi Arabia \\ ${ }^{3}$ Department of Mathematics, College of Arts and Sciences, Wadi Aldawaser, Prince Sattam bin \\ Abdulaziz University, Saudi Arabia
}

*Corresponding author: n.sooppy@psau.edu.sa; ksnisar1@gmail.com

\begin{abstract}
In this article, our aim is to consider a class of nonconvex fuzzy mapping known as exponentially preinvex fuzzy mapping. With the support of some examples, the notions of exponentially preinvex fuzzy mappings are explored and discussed in some special cases. Some properties are also derived and relations among the exponentially preinvex fuzzy mappings (exponentially preinvex-FMs), exponentially invex fuzzy mappings (exponentially-IFMs), and exponentially monotonicity are established under some mild conditions. In the end, using the fact that fuzzy optimization and fuzzy variational inequalities have close relationships, we have proven that the optimality conditions of exponentially preinvex fuzzy mapping can be distinguished by exponentially fuzzy variational-like inequality and exponentially fuzzy mixed variational-like inequality. These inequalities render the very interesting outcomes of our main results and appear to be the new ones. Presented results in this paper can be considered and the development of previously obtained results.
\end{abstract}

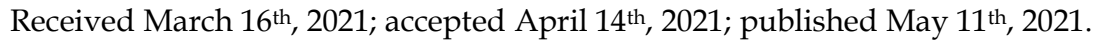

2010 Mathematics Subject Classification. 49J40.

Key words and phrases. exponentially preinvex fuzzy mapping; exponentially invex fuzzy mappings; exponentially monotone fuzzy mappings; exponentially fuzzy mixed variational-like inequalities.

(C)2021 Authors retain the copyrights of their papers, and all open access articles are distributed under the terms of the Creative Commons Attribution License. 


\section{INTRODUCTION}

In the last few decades, the ideas of convexity and nonconvexity are well-acknowledged in optimization concepts and gifted a vital role in operation research, economics, decision making, and management. Hanson [16] initiated to introduce a generalized class of convexity which is known as an invex function. The invex function played a significant role in mathematical programming. A step forward, the invex set invex function and preinvex function were introduced and studied by Israel and Mond [9], and Mohen and Neogy [20]. Also, Noor [23] examined the optimality conditions of differentiable preinvex functions and proved that variational-like inequalities would characterize the minimum. Many classical convexity generalizations and extensions have been investigated by several authors, see ([1], [6], [7], [15]) and the references therein. It is well known that logarithmically convex functions have serious importance in convex theory because by using these functions we can derive more accurate inequalities as compare to convex functions. Some of the authors discussed different classes of convex and nonconvex functions. These functions have a very close relationship with logarithmically convex functions. Bernstain [10], initiated to introduce exponentially convex function. A move forward, the concept of an r-convex function was introduced by Avriel [5], while Antczak [4] studied the notions of $(r, p)$-convex functions. For further details on r-convex function, we refer the reader to study the properties of r-convex function, see [34] and the reference therein. Exponentially convex functions and their generalizations have many applications in different fields such as information theory, data analysis, statistics, and machine learning; see [2-5]. Noor and Noor [25-27] have recently considered and characterized another type of exponentially convex functions and characterized its properties, and have demonstrated that convex functions are different from the exponentially convex functions defined by Bernstein [10]. Furthermore, Noor and Noor [28] generalized the exponentially convex functions and defined a class of nonconvex functions which is known as exponentially preinvex function and proved that the minimum of a differentiable exponentially convex function is distinguished by exponentially variational-like inequality.

Similarly, the notions of convexity and nonconvexity play a vital role in optimization under

fuzzy domain because, during characterization of the optimality condition of convexity, we obtain fuzzy variational inequalities so variational inequality theory and fuzzy complementary 
problem theory established powerful mechanism of the mathematical problems and they have a friendly relationship. Many authors contributed to this fascinating and interesting field. The concept of fuzzy mappings (FMs) was proposed by Chang and Zadeh [11] in 1972. In 1989, Nanda and Kar [21] were initiated to introduce convex-FMs and characterized the notion of convex-FM through the idea of epigraph. A step forward, Furukawa [14] and Syau [31] proposed and examined FM from space $\mathbb{R}^{n}$ to the set of fuzzy numbers, fuzzy valued Lipschitz continuity, logarithmic convex-FMs and quasi-convex-FMs. Besides, Chang [12] discussed the idea of convex-FM and find its optimality condition with the support of fuzzy variational inequality. Generalization and extension of fuzzy convexity play a vital and significant implementation in diverse directions. So let's note that, one of the most considered classes of nonconvex-FM is preinvex-FM. Noor [22] introduced this idea and proved some results that distinguish the fuzzy optimality condition of differentiable fuzzy preinvex mappings by fuzzy variational-like inequality. Fuzzy variational inequality theory and complementary problem theory established a strong relationship with mathematical problems. Recently, Li and Noor [19] established an equivalence condition of preinvex-FM and characterizations about preinvex-FMs under some mild conditions. With the support of examples, $\mathrm{Wu}$ and $\mathrm{Xu}$ [33] updated the definition of convex-FMs and established a new approach regarding the existence of a fuzzy preinvex mapping under the condition of lower or upper semicontinuity. In 2012, RufianLizana et al. [29] reviewed the existing literature and made appropriate modifications to the results obtained by $\mathrm{Wu}$ and $\mathrm{Xu}$ [32] regarding invex-FMs. For differentiable and twice differentiable preinvex-FMs, Rufian-Lizana et al. [30] gave the required and sufficient conditions. They demonstrated the validity of characterizations with the help of examples and improved the previous results provided by Li and Noor [19]. We refer to the readers for further analysis of literature on the applications and properties of variational-like inequalities and generalized convex-FMs, see ([8], [13], [15], [24], [32]) and the references therein.

Motivated and inspired by the ongoing research work, we note that convex and generalized convex-FMs play an important role in fuzzy optimization. The paper is organized as follows. Section 2 recalls some basic and new definitions, preliminary notations, and new results which will be helpful for further study. Section 3 introduces and considers a family of classes of nonconvex-FMs is called exponentially preinvex-FMs and investigates some 
properties. Section 4 derives some relations among the exponentially preinvex-FMs, exponentially-IFMs, and exponentially monotonicity under some mild conditions. Section 5 introduces the new classes of fuzzy variational-like inequality which is known as exponentially fuzzy variational-like inequality and exponentially fuzzy mixed variational-like inequality. Several special cases are also discussed. These inequalities give an interesting outcome of our main results.

\section{PRELIMINARIES}

Let $\mathbb{R}$ be the set of real numbers. A fuzzy subset set $\mathcal{A}$ of $\mathbb{R}$ is distinguished by a function $\varphi: \mathbb{R} \rightarrow[0,1]$ called the membership function. In this study this depiction is approved. Moreover, the collection of all fuzzy subsets of $\mathbb{R}$ is denoted by $\widetilde{\mathbb{F}}(\mathbb{R})$.

Definition 2.1. If $\varphi$ be a fuzzy set in $\mathbb{R}$ and $\gamma \in[0,1]$, then $\gamma$-level sets of $\varphi$ is denoted and is defined as follows

$$
\varphi_{\gamma}=\{u \in \mathbb{R} \mid \varphi(u) \geq \gamma\}
$$

Definition 2.2. A fuzzy number $(\mathrm{FN}) \varphi$ is a fuzzy set in $\mathbb{R}$ with the following properties:

(1) $\varphi$ is normal i.e. there exists $x \in \mathbb{R}$ such that $\varphi(x)=1$;

(2) $\varphi$ is upper semi continuous i.e., for given $x \in \mathbb{R}$, for every $x \in \mathbb{R}$ there exist $\varepsilon>0$ there exist $\delta>0$ such that $\varphi(x)-\varphi(y)<\varepsilon$ for all $y \in \mathbb{R}$ with $|x-y|<\delta$.

(3) $\varphi$ is fuzzy convex i.e., $\varphi((1-\tau) x+\tau y) \geq \min (\varphi(x), \varphi(y)), \forall x, y \in \mathbb{R}$ and $\tau \in[0,1]$;

(4) $\varphi$ is compactly supported i.e., $\operatorname{cl}\{x \in \mathbb{R} \mid \varphi(x)>0\}$ is compact.

The LR-FNs first introduced by Dubois and Prade [13] are defined as follows:

Definition 2.3. Let $L, R:[0,1] \rightarrow[0,1]$ be two decreasing and upper semicontinuous functions with $L(0)=R(0)=1$ and $L(1)=R(1)=0$. Then the FN is defined as

$$
\begin{gathered}
\varphi(u)= \\
\left\{\begin{array}{cc}
L\left(\frac{c-u}{\rho}\right) & c-\rho \leq u<d, \\
1 & c \leq u<d, \\
L\left(\frac{u-d}{r}\right) & c \leq u<d+r, \\
0 & \text { otherwise }
\end{array}\right.
\end{gathered}
$$

where $r, \rho>0$ and $d \geq c$. 
Let $\mathbb{F}(\mathbb{R})$ denotes the set of all FNs and let $\varphi \in \mathbb{F}(\mathbb{R})$ be $F N$, if and only if, $\gamma$-levels $\varphi_{\gamma}$ is a nonempty compact convex set of $\mathbb{R}$. This is represented by

$$
\varphi_{\gamma}=\left[\varphi_{*}(\gamma), \varphi^{*}(\gamma)\right]
$$

where

$$
\varphi_{*}(\gamma)=\inf \{u \in \mathbb{R} \mid \varphi(u) \geq \gamma\}, \varphi^{*}(\gamma)=\sup \{u \in \mathbb{R} \mid \varphi(u) \geq \gamma\} .
$$

Since each $\rho \in \mathbb{R}$ is also a FN, defined as

$$
\tilde{\rho}(u)=\left\{\begin{array}{l}
1 \text { if } u=\rho \\
0 \text { if } u \neq \rho
\end{array}\right.
$$

It is well known that a FN is a real fuzzy interval and $\varphi$ can also be identified by a triplet:

$$
\left\{\left(\varphi_{*}(\gamma), \varphi^{*}(\gamma), \gamma\right): \gamma \in[0,1]\right\} .
$$

This notion help us to characterize a FN in terms of the two end point functions $\varphi_{*}(\gamma)$ and $\varphi^{*}(\gamma)$

Theorem $2.1([13])$. Suppose that $\varphi_{*}(\gamma):[0,1] \rightarrow \mathbb{R}$ and $\varphi^{*}(\gamma):[0,1] \rightarrow \mathbb{R}$ satisfy the following assertions:

- $\varphi_{*}(\gamma)$ is a non-decreasing function.

- $\varphi^{*}(\gamma)$ is a non-increasing function.

- $\varphi_{*}(1) \leq \varphi^{*}(1)$.

- $\varphi_{*}(\gamma)$ and $\varphi^{*}(\gamma)$ are bounded and left continuous on $(0,1]$ and right continuous at $\gamma=0$.

Then $\varphi: \mathbb{R} \rightarrow[0,1]$, defined by

$$
\varphi(u)=\sup \left\{\gamma: \varphi_{*}(\gamma) \leq u \leq \varphi^{*}(\gamma)\right\}
$$

is a $\mathrm{FN}$, parameterization is given by $\left\{\left(\varphi_{*}(\gamma), \varphi^{*}(\gamma), \gamma\right): \gamma \in[0,1]\right\}$. Moreover, if $\varphi: \mathbb{R} \rightarrow[0,1]$ is a FN with parametrization given by $\left\{\left(\varphi_{*}(\gamma), \varphi^{*}(\gamma), \gamma\right): \gamma \in[0,1]\right\}$, then function $\varphi_{*}(\gamma)$ and $\varphi^{*}(\gamma)$ find the conditions (1)-(4).

Let $\varphi, \phi \in \mathbb{F}(\mathbb{R}) \quad$ represented parametrically $\left\{\left(\varphi_{*}(\gamma), \varphi^{*}(\gamma), \gamma\right): \gamma \in[0,1]\right\} \quad$ and $\left\{\left(\varphi_{*}(\gamma), \phi^{*}(\gamma), \gamma\right): \gamma \in[0,1]\right\}$ respectively. We say that $\varphi \leq \phi$ if for all $\gamma \in(0,1], \varphi^{*}(\gamma) \leq \phi^{*}(\gamma)$, and $\varphi_{*}(\gamma) \leq \phi_{*}(\gamma)$. If $\varphi \leq \phi$, then there exist $\gamma \in(0,1]$ such that $\varphi^{*}(\gamma)<\phi^{*}(\gamma)$ or $\varphi_{*}(\gamma) \leq$ $\phi_{*}(\gamma)$. We say comparable if for any $\varphi, \phi \in \mathbb{F}(\mathbb{R})$, we have $\varphi \leq \phi$ or $\varphi \geq \phi$ otherwise they are non-comparable. Some time we may write $\varphi \preceq \phi$ instead of $\phi \geq \varphi$ and note that, we may say that $\mathbb{F}(\mathbb{R})$ is a partial ordered set under the relation $\leq$. 
If $\varphi, \phi \in \mathbb{F}(\mathbb{R})$, there exist $\psi \in \mathbb{F}(\mathbb{R})$ such that $\varphi=\phi \widetilde{+} \psi$, then by this result we have existence of Hukuhara difference of $\varphi$ and $\phi$, and we say that $\psi$ is the H-difference of $\varphi$ and $\phi$, and denoted by $\varphi \simeq \phi$, see [32]. If H-difference exists, then

$$
(\psi)^{*}(\gamma)=(\varphi \simeq \phi)^{*}(\gamma)=\varphi^{*}(\gamma)-\phi^{*}(\gamma),(\psi)_{*}(\gamma)=(\varphi \simeq \phi)_{*}(\gamma)=\varphi_{*}(\gamma)-\phi_{*}(\gamma) .
$$

Now we discuss some properties of FNs under addition and scalar multiplication, if $\varphi, \phi \in \mathbb{F}(\mathbb{R})$ and $\rho \in \mathbb{R}$ then $\varphi \widetilde{+} \phi$ and $\rho \varphi$ define as

$$
\begin{aligned}
\varphi \widetilde{+} \phi & =\left\{\left(\varphi_{*}(\gamma)+\phi_{*}(\gamma), \varphi^{*}(\gamma)+\phi^{*}(\gamma), \gamma\right): \gamma \in[0,1]\right\}, \\
\rho \varphi & =\left\{\left(\rho \varphi_{*}(\gamma), \rho \varphi^{*}(\gamma), \gamma\right): \gamma \in[0,1]\right\} .
\end{aligned}
$$

Remark 2.1. Obviously, $\mathbb{F}(\mathbb{R})$ is closed under addition and nonnegative scaler multiplication and above defined properties on $\mathbb{F}(\mathbb{R})$ are equivalent to those derived from the usual extension principle. Furthermore, for each scalar number $\rho \in \mathbb{R}$,

$$
\varphi \widetilde{+} \rho=\left\{\left(\varphi_{*}(\gamma)+\rho, \varphi^{*}(\gamma)+\rho, \gamma\right): \gamma \in[0,1]\right\} .
$$

Definition 2.4. A mapping $\mathcal{T}: K \subset \mathbb{R} \rightarrow \mathbb{F}(\mathbb{R})$ is called fuzzy mapping (FM). For each $\gamma \in[0,1]$, denote $[\mathcal{T}(u)]^{\gamma}=\left[\mathcal{T}_{*}(u, \gamma), \mathcal{T}^{*}(u, \gamma)\right]$. Thus a FM $\mathcal{T}$ can be identified by a parametrized triples

$$
\mathcal{T}(u)=\left\{\left(\mathcal{T}_{*}(u, \gamma), \mathcal{T}^{*}(u, \gamma), \gamma\right): \gamma \in[0,1]\right\} .
$$

Definition 2.5. Let $\mathcal{T}: K \subset \mathbb{R} \rightarrow \mathbb{F}(\mathbb{R})$ be a FM. Then $\mathcal{T}(u)$ is said to be continuous at $u \in K$, if for each $\gamma \in[0,1]$, both end point functions $\mathcal{T}_{*}(u, \gamma)$ and $\mathcal{T}^{*}(u, \gamma)$ are continuous at $u \in K$.

Definition 2.6 ([8]). Let $L=(m, n)$ and $u \in L$. Then $\mathrm{FM} \mathcal{T}:(m, n) \rightarrow \mathbb{F}(\mathbb{R})$ is said to be a generalized differentiable (in short, G-differentiable) at $u$ if there exists an element $\mathcal{T}(u) \in$ $\mathbb{F}(\mathbb{R})$ such that

for all $0<\tau$, sufficiently small, there exist $\mathcal{T}(u+\tau) \simeq \mathcal{T}(u), \mathcal{T}(u) \simeq \mathcal{T}(u-\tau)$ and the limits

$$
\begin{aligned}
& \lim _{\tau \rightarrow 0^{+}} \frac{\mathcal{T}(u+\tau) \simeq \mathcal{T}(u)}{\tau}=\lim _{\tau \rightarrow 0^{+}} \frac{\mathcal{T}(u) \simeq \mathcal{T}(u-\tau)}{\tau}=\mathcal{T}^{\prime}(u) \text { or } \lim _{\tau \rightarrow 0^{+}} \frac{\mathcal{T}(u) \simeq \mathcal{T}(u+\tau)}{-\tau}=\lim _{\tau \rightarrow 0^{+}} \frac{\mathcal{T}(u-\tau) \simeq \mathcal{T}(u)}{-\tau}=\mathcal{T}^{\prime}(u) \text { or } \\
& \lim _{\tau \rightarrow 0^{+}} \frac{\mathcal{T}(u+\tau) \simeq \mathcal{T}(u)}{\tau}=\lim _{\tau \rightarrow 0^{+}} \frac{\mathcal{T}(u-\tau) \simeq \mathcal{T}(u)}{-\tau}=\mathcal{T}^{\prime}(u) \text { or } \lim _{\tau \rightarrow 0^{+}} \frac{\mathcal{T}(u) \simeq \mathcal{T}(u+\tau)}{-\tau}=\lim _{\tau \rightarrow 0^{+}} \frac{\mathcal{T}(u) \simeq \mathcal{T}(u-\tau)}{\tau}=\mathcal{T}^{\prime}(u),
\end{aligned}
$$

where the limits are taken in the metric space $(\mathbb{F}(\mathbb{R}), D)$, for $\varphi, \phi \in \mathbb{F}(\mathbb{R})$

$$
D(\varphi, \phi)=\sup _{0 \leq \gamma \leq 1} H\left(\varphi_{\gamma}, \phi_{\gamma}\right),
$$

and $H$ denote the well-known Hausdorff metric on space of intervals.

Definition 2.7 ([21]). A FM $\mathcal{T}: K \rightarrow \mathbb{F}(\mathbb{R})$ is called convex on the convex set $K$ if

$$
\mathcal{T}((1-\tau) u+\tau \vartheta) \leq(1-\tau) \mathcal{T}(u) \widetilde{+} \tau \mathcal{T}(\vartheta), \forall u, \vartheta \in K, \tau \in[0,1] .
$$

Definition 2.8 ([21]). A FM $\mathcal{T}: K \rightarrow \mathbb{F}(\mathbb{R})$ is called quasi-convex on the convex set $K$ if 


$$
\mathcal{T}((1-\tau) u+\tau \vartheta) \leq \max (\mathcal{T}(u), \mathcal{T}(\vartheta)), \forall u, \vartheta \in K, \tau \in[0,1] .
$$

Definition $2.9([22])$. A FM $\mathcal{T}: K_{\partial} \rightarrow \mathbb{F}(\mathbb{R})$ is called preinvex-FM on the invex set $K_{\partial}$ w.r.t. bifunction $\partial$ if

$$
\mathcal{T}(u+\tau \partial(\vartheta, u)) \leq(1-\tau) \mathcal{T}(u) \widetilde{+} \tau(\vartheta), \forall u, \vartheta \in K_{\partial}, \tau \in[0,1],
$$

where $\partial: K_{\partial} \times K_{\partial} \rightarrow \mathbb{R}$.

Lemma 2.1 ([19]). Let $K_{\partial}$ be an invex set w.r.t. $\partial$ and let $\mathcal{T}: K_{\partial} \rightarrow \mathbb{F}(\mathbb{R})$ be a FM parametrized by

$$
\mathcal{T}(u)=\left\{\left(\mathcal{T}_{*}(u, \gamma), \mathcal{T}^{*}(u, \gamma), \gamma\right): \gamma \in[0,1]\right\}, \forall u \in K_{\partial} .
$$

Then $\mathcal{T}$ is preinvex-FM on $K_{\partial}$ if and only if, for all $\gamma \in[0,1], \mathcal{T}_{*}(u, \gamma)$ and $\mathcal{T}^{*}(u, \gamma)$ are preinvex functions w.r.t. $\partial$ on $K_{\partial}$.

Definition 2.10 ([22]). A comparable $\mathrm{FM} \mathcal{T}: K_{\partial} \rightarrow \mathbb{F}(\mathbb{R})$ is called quasi-preinvex-FM on the invex set $K_{\partial}$ w.r.t. $\partial$ if

$$
\mathcal{T}(u+\tau \partial(\vartheta, u)) \leq \max (\mathcal{T}(u), \mathcal{T}(\vartheta)), \forall u, \vartheta \in K_{\partial}, \tau \in[0,1] .
$$

Definition 2.11 ([28]). A function $\mathcal{T}: K \rightarrow \mathbb{R}$ is called exponentially convex on $K$, if

$$
e^{\mathcal{T}((1-\tau) u+\tau \vartheta)} \leq(1-\tau) e^{\mathcal{T}(u)}+\tau e^{\mathcal{T}(\vartheta)}, \forall u, \vartheta \in K, \tau \in[0,1]
$$

The Definition 2.11, can also be written in the following equivalent way, which is due to Antczak [4].

Definition 2.12. A function $\mathcal{T}: K \rightarrow \mathbb{R}$ is called exponentially convex on $K$, if

$$
\mathcal{T}((1-\tau) u+\tau \vartheta) \leq \log \left[(1-\tau) e^{\mathcal{T}(u)}+\tau e^{\mathcal{T}(\vartheta)}\right], \forall u, \vartheta \in K, \tau \in[0,1] .
$$

Strictly exponentially convex function if strict inequality holds for $\mathcal{T}(u) \neq \mathcal{T}(\vartheta) . \mathcal{T}: K \rightarrow \mathbb{F}_{0}$ is called exponentially concave function if $-\mathcal{T}$ is exponentially convex on $K$. Strictly exponentially concave function if strict inequality holds for $\mathcal{T}(u) \neq \mathcal{T}(\vartheta)$.

Antczak [4] and Alirezaei and Mathar [3 have discussed the applications of exponentially convex function in the mathematical programing and information theory. Similarly, Alirezaei and Mazhar [3] explore the applications of exponentially concave function with following example in communication and information theory.

Example. The error function

$$
\operatorname{erf}(u)=\frac{2}{\sqrt{\Pi}} \int_{0}^{u} e^{-t^{2}} d u
$$

becomes an exponentially concave function in the form $\operatorname{erf}(\sqrt{u}), u \geq 0$, which defines the bit/symbol error probability of communication system depending on the square root of the 
underlying signal-to-noise ratio. This demonstrates that the exponentially concave functions in the theory communication and information theory may play an important role.

Definition 2.13 ([28]). A function $\mathcal{T}: K \rightarrow \mathbb{R}$ is called exponentially quasi convex on $K$, if

$$
e^{\mathcal{T}((1-\tau) u+\tau \vartheta)} \preceq \max \left(e^{\mathcal{T}(u)}, e^{\mathcal{T}(\vartheta)}\right), \forall u, \vartheta \in K, \tau \in[0,1] .
$$

From above definitions we can easily prove that each exponentially convex function is exponentially quasi convex function but the converse is not true such that

$$
\begin{aligned}
e^{\mathcal{T}((1-\tau) u+\tau \vartheta)} & \leq(1-\tau) e^{\mathcal{T}(u)}+\tau e^{\mathcal{T}(\vartheta)}, \\
& \leq \max \left(e^{\mathcal{T}(u)}, e^{\mathcal{T}(\vartheta)}\right) .
\end{aligned}
$$

Definition 2.14. Let $K_{\partial}$ be an invex set. Then FM $\mathcal{T}: K_{\partial} \rightarrow \mathbb{F}_{0}$ is said to be:

- $\quad$ exponentially preinvex-FM (exponentially preinvex-FM) on $K_{\partial}$ w.r.t. bi-function $\partial(.,$.$) if$

$$
e^{\mathcal{T}(u+\tau \partial(\vartheta, u))} \preceq(1-\tau) e^{\mathcal{T}(u)} \widetilde{+} \tau e^{\mathcal{T}(\vartheta)}, \forall \vartheta \in K_{\partial}, \tau \in[0,1] .
$$

- $\quad$ exponentially preconcave-FM on $K_{\partial}$ if inequality (2.5) is reversed.

- $\quad$ strictly exponentially preinvex-FM on $K_{\partial}$ w.r.t. bi-function $\partial(.,$.$) if$

$$
e^{\mathcal{T}(u+\tau \partial(\vartheta, u))} \prec(1-\tau) e^{\mathcal{T}(u)} \widetilde{\mp} \tau e^{\mathcal{T}(\vartheta)}, \forall \vartheta \in K_{\partial}, \tau \in[0,1] .
$$

- $\quad$ strictly exponentially preconcave-FM on $K_{\partial}$ if inequality (2.6) is reversed.

\section{Remark 2.2.}

(i) If $\mathcal{T}$ is exponentially preinvex-FM, then $\lambda \mathcal{T}$ is also exponentially preinvex-FM for $\lambda \geq$ 0.

(ii) If $\mathcal{T}(u)$ and $G(u)$ both are exponentially preinvex-FMs, then $\max (\mathcal{T}(u), G(u))$ is also exponentially preinvex-FMs.

Special cases

(iii) The exponentially preinvexity of $\mathcal{T}$ on $K_{\partial}$ is equivalent to preinvexity of $e^{\mathcal{T}(u)}$.

(iv) The exponentially prconcavity of $\mathcal{T}$ on $K_{\partial}$ is equivalent to preconcavety of $e^{\mathcal{T}(u)}$.

(v) By using remark (iii), if $\partial(\vartheta, u)=\vartheta-u$, then exponentially preinvex-FM becomes exponentially convex-FM, that is

$$
e^{\mathcal{T}(u+\tau(\vartheta-u))} \preceq(1-\tau) e^{\mathcal{T}(u)} \widetilde{+} \tau e^{\mathcal{T}(\vartheta)}, \forall u, \vartheta \in K_{\partial}, \tau \in[0,1] .
$$

(vi) If $\tau=\frac{1}{2}$, then (2.5) becomes

$$
e^{\mathcal{T}\left(\frac{2 u+\partial(\vartheta, u)}{2}\right)} \preceq \frac{e^{\mathcal{T}(u)} \widetilde{+} e^{\mathcal{T}(\vartheta)}}{2}, \forall u, \vartheta \in K_{\partial} .
$$


The inequality (2.7) is known as exponentially Jenson preinvex-FMs.

We can easily discuss the next result with the help of Remark 2.2 (iii).

Theorem 2.2. Let $\mathrm{K}_{\partial}$ be an invex set w.r.t. $\partial$ and let $\mathcal{T}: \mathrm{K}_{\partial} \rightarrow \mathbb{F}_{0}$ be a FM parameterized by

$$
\mathcal{T}(u)=\left\{\left(\mathcal{T}_{*}(u, \gamma), \mathcal{T}^{*}(u, \gamma), \gamma\right): \gamma \in[0,1]\right\}, \forall u \in \mathrm{K}_{\partial} .
$$

Then $\mathcal{T}$ is exponentially preinvex-FM on $\mathrm{K}_{\partial}$ if and only if, for all $\gamma \in[0,1]$,

$\mathcal{T}_{*}(u, \gamma)$ and $\mathcal{T}^{*}(u, \gamma)$ are exponentially preinvex function w.r.t. $\partial$.

Proof. The demonstration of proof is similar to Lemma 2.1, by Remark 2.2 (iii).

Example 2.1. We consider the FMs $\mathcal{T}:(-1,1) \rightarrow \mathbb{F}_{0}$ defined by,

$$
\mathcal{T}(u)(\sigma)=\left\{\begin{array}{cc}
\frac{\sigma-u^{2}}{1-u^{2}} & \sigma \in\left[u^{2}, 1\right), \\
0 & \text { otherwise. }
\end{array}\right.
$$

Then, for each $\gamma \in[0,1]$, we have $\mathcal{T}_{\gamma}(u)=\left[\gamma+(1-\gamma) u^{2}, 1\right]$. Since end point functions $\mathcal{J}_{*}(\gamma)$, $\mathcal{T}^{*}(\gamma)$ are exponentially preinvex functions for each $\gamma \in[0,1]$, then $\mathcal{T}$ is exponentially preinvexFM w.r.t. bi-function

$$
\partial(\vartheta, u)=\left\{\begin{array}{cl}
\frac{\vartheta^{2}-u^{2}}{2 u} & \vartheta^{2}-u^{2}<0, \\
0 & \text { otherwise. }
\end{array}\right.
$$

Example 2.2. We consider the FMs $\mathcal{T}:(0, \infty) \rightarrow \mathbb{F}_{0}$ defined by,

$$
\mathcal{T}(u)(\sigma)=\left\{\begin{array}{cl}
\frac{\sigma}{u^{2}} & \sigma \in\left[0, u^{2}\right], \\
\frac{2 u^{2}-\sigma}{u^{2}} & \sigma \in\left(u^{2}, 2 u^{2}\right], \\
0 & \text { otherwise. }
\end{array}\right.
$$

Then, for each $\gamma \in[0,1]$, we have $\mathcal{T}_{\gamma}(u)=\left[\gamma u^{2},(2-\gamma) u^{2}\right]$. Since end point functions $\mathcal{T}_{*}(\gamma)$, $\mathcal{T}^{*}(\gamma)$ are exponentially preinvex functions for each $\gamma \in[0,1]$, then $\mathcal{T}$ is exponentially preinvexFM w.r.t.

$$
\partial(\vartheta, u)=\vartheta-u
$$

Definition 2.15. A comparable $\mathrm{FM} \mathcal{T}: K_{\partial} \rightarrow \mathbb{F}_{0}$ is said to be:

- $\quad$ exponentially quasi-preinvex-FM on $K_{\partial}$ w.r.t. bi-function $\partial(.,$.$) , if$

$$
e^{\mathcal{T}(u+\tau \partial(\vartheta, u))} \preceq \max \left(e^{\mathcal{T}(u)}, e^{\mathcal{T}(\vartheta)}\right), \forall \vartheta \in K_{\partial}, \tau \in[0,1] .
$$

- $\quad$ exponentially quasi-preconcave-FM on $K_{\partial}$ if inequality (2.8) is reversed.

- $\quad$ strictly exponentially quasi-preinvex-FM on $K_{\partial}$ w.r.t. bi-function $\partial(.,$.$) , if$

$$
e^{\mathcal{T}(u+\tau \partial(\vartheta, u))} \prec \max \left(e^{\mathcal{T}(u)}, e^{\mathcal{T}(\vartheta)}\right), \forall \vartheta \in K_{\partial}, \tau \in[0,1] .
$$


- strictly exponentially quasi-preconcave-FM on $K_{\partial}$ if inequality (2.9) is reversed.

Proposition 2.1. Let comparable $\mathrm{FM} \mathcal{T}: K_{\partial} \rightarrow \mathbb{F}_{0}$ be an exponentially preinvex-FM, such that $\mathcal{T}(\vartheta) \prec \mathcal{T}(u)$. Then $\mathcal{T}$ is strictly exponentially quasi-preinvex-FM.

Proof. Let $e^{\mathcal{T}(\vartheta)} \prec e^{\mathcal{T}(u)}$ and $\mathcal{T}$ be exponentially preinvex-FM. Then, for all $u, \vartheta \in K_{\partial}$ and $\tau \in$ $[0,1]$ we have

$$
e^{\mathcal{T}(u+\tau \partial(\vartheta, u))} \preceq(1-\tau) e^{\mathcal{T}(u)} \widetilde{+} \tau e^{\mathcal{T}(\vartheta)},
$$

Since $\mathcal{T}(\vartheta) \prec \mathcal{T}(u)$, we have

$$
e^{\mathcal{T}(u+\tau \partial(\vartheta, u))} \prec e^{\mathcal{T}(u)}=\max \left(e^{\mathcal{T}(u)}, e^{\mathcal{T}(\vartheta)}\right) .
$$

Hence, $\mathcal{T}$ is strictly exponentially quasi-preinvex-FM.

Definition 2.16. A FM $\mathcal{T}: K_{\partial} \rightarrow \mathbb{F}_{0}$ is said to be:

- $\quad$ exponentially logarithmic preinvex-FM on $K_{\partial}$ w.r.t. bi-function $\partial(.,$.$) , if$

$$
e^{\mathcal{T}(u+\tau \partial(\vartheta, u))} \leq\left(e^{\mathcal{T}(u)}\right)^{1-\tau}\left(e^{\mathcal{T}(\vartheta)}\right)^{\tau}, \forall \vartheta \in K_{\partial}, \tau \in[0,1] .
$$

- exponentially logarithmic preconcave-FM on $K_{\partial}$ if inequality (2.10) is reversed.

- strictly exponentially logarithmic preinvex-FM on $K_{\partial}$ w.r.t. bi-function $\partial(.$, . ), if

$$
e^{\mathcal{T}(u+\tau \partial(\vartheta, u))} \prec\left(e^{\mathcal{T}(u)}\right)^{1-\tau}\left(e^{\mathcal{T}(\vartheta)}\right)^{\tau}, \forall \vartheta \in K_{\partial}, \tau \in[0,1] .
$$

- strictly exponentially logarithmic preconcave-FM on $K_{\partial}$ if inequality (2.11) is reversed, where $\mathcal{T}()>.\widetilde{0}$.

For further study, let $K_{\partial}$ be a nonempty closed invex set in $\mathbb{R}$. Let $\mathcal{T}: K_{\partial} \rightarrow \mathbb{F}(\mathbb{R})$ be a FM and $\partial: K_{\partial} \times K_{\partial} \rightarrow \mathbb{R}$ be an arbitrary bifunction. We denote $\langle.,$.$\rangle be the inner product.$

Next, we will discuss exponentially preinvex-FMs by using Remark 2.2, (iii).

\section{BASIC RESULTS}

In this section, we investigate some basic properties of exponentially preinvex-FMs and obtain some characterization for exponentially preinvex-FMs by using the concepts of level sets and epigraph of FMs.

Definition 3.1. Let $\mathcal{T}: \mathcal{D} \rightarrow \mathbb{F}_{0}$ and $G: \mathcal{D} \rightarrow \mathbb{F}_{0}$ be two FMs on $\mathcal{D} \subseteq \mathbb{R}$. Then $\mathcal{T}(u)$ and $G(u)$ are said to be fuzzy comonotonic on $\mathcal{D}$ if, for all $u, \vartheta \in \mathcal{D}$,

$$
\left[e^{\mathcal{T}(u)} \simeq e^{\mathcal{T}(\vartheta)}\right]\left[e^{G(u)} \simeq e^{G(\vartheta)}\right] \geq \tilde{0} .
$$


Theorem 3.1. Let $\mathcal{T}(u)$ and $G(u)$ be two exponentially preinvex-FMs on $K_{\partial}$. Then $\mathcal{T}(u)=$ $\mathcal{T}(u) \widetilde{+} G(u)$ is exponentially preinvex-FM on $K_{\partial}$, provide that $\mathcal{T}(u)$ and $G(u)$ are fuzzy comonotonic on $K_{\partial}$.

Proof. Let $\mathcal{T}(u)$ and $G(u)$ be two exponentially preinvex-FMs on $K_{\partial}$. Then, for all $u, \vartheta \in K_{\partial}$ and $\tau \in[0,1]$, we have

$$
\begin{aligned}
& e^{\mathcal{T}(u+\tau \partial(\vartheta, u))} \preceq(1-\tau) e^{\mathcal{T}(u)} \widetilde{\mp} \tau e^{\mathcal{T}(\vartheta)}, \\
& e^{G(u+\tau \partial(\vartheta, u))} \preceq(1-\tau) e^{G(u)} \widetilde{\mp} \tau e^{G(\vartheta)} .
\end{aligned}
$$

Now,

$$
\begin{aligned}
& e^{\mathcal{T}(u+\tau \partial(\vartheta, u)) \widetilde{+} G(u+\tau \partial(\vartheta, u))}=e^{\mathcal{T}(u+\tau \partial(\vartheta, u))} e^{G(u+\tau \partial(\vartheta, u))}, \\
& \leq\left[(1-\tau) e^{\mathcal{T}(u)} \widetilde{\mp} \tau e^{\mathcal{T}(\vartheta)}\right]\left[(1-\tau) e^{G(u)} \widetilde{\mp} \tau e^{G(\vartheta)}\right], \\
& =(1-\tau)^{2} e^{\mathcal{T}(u) \widetilde{+} G(u)} \widetilde{+}(1-\tau) \tau\left[e^{\mathcal{T}(u) \widetilde{+} G(\vartheta)} \widetilde{+} e^{\mathcal{T}(\vartheta) \widetilde{+} G(u)}\right] \\
& \widetilde{+} \tau^{2} e^{\mathcal{T}(\vartheta) \widetilde{\Psi} G(\vartheta) .}
\end{aligned}
$$

Since $\mathcal{T}(u)$ and $G(u)$ are fuzzy comonotonic on $K_{\partial}$, such that

$$
\left[e^{\mathcal{T}(u)} \simeq e^{\mathcal{T}(\vartheta)}\right]\left[e^{G(u)} \simeq e^{G(\vartheta)}\right] \geq \tilde{0},
$$

which implies that

$$
e^{\mathcal{T}(u) \widetilde{+} G(u)} \widetilde{\mp} e^{\mathcal{T}(\vartheta) \widetilde{+} G(\vartheta)} \geq e^{\mathcal{T}(u) \widetilde{+} G(\vartheta)} \widetilde{\mp} e^{\mathcal{T}(\vartheta) \widetilde{\Psi} G(u)} .
$$

It follows by substituting (3.4) into (3.3) that

$$
\begin{aligned}
e^{\mathcal{T}(u+\tau \partial(\vartheta, u)) \widetilde{\mp}(u+\tau \partial(\vartheta, u)) \leq} & (1-\tau)^{2} e^{\mathcal{T}(u) \widetilde{+} G(u)}+(1-\tau) \tau\left[e^{\mathcal{T}(u) \widetilde{+} G(u)} \widetilde{\mp} e^{\mathcal{T}(\vartheta) \widetilde{\mp} G(\vartheta)}\right] \\
& \widetilde{\mp} \tau^{2} e^{\mathcal{T}(\vartheta) \widetilde{+} G(\vartheta)}, \\
= & (1-\tau) e^{\mathcal{T}(u) \widetilde{+} G(u)} \widetilde{+} \tau e^{\mathcal{T}(\vartheta) \widetilde{\mp} G(\vartheta) .}
\end{aligned}
$$

Hence, $\mathcal{T}(u) \widetilde{f} G(u)$ is exponentially preinvex-FM on $K_{\partial}$.

Theorem 3.2. Let $\mathcal{T}: K_{\partial} \rightarrow \mathbb{F}_{0}$ be a FM, with $\theta=\inf _{u \in K_{\partial}}\left(e^{\mathcal{T}(u)}\right)$ exists in $\mathbb{F}_{0}$.

(1) If $\mathcal{T}: K_{\partial} \rightarrow \mathbb{F}_{0}$ is an exponentially preinvex-FM on $K_{\partial}$, then set $\Omega=\left\{u: u \in K_{\partial}, e^{\mathcal{T}(u)}=\theta\right\}$ is an invex set.

(2) If $\mathcal{T}: K_{\partial} \rightarrow \mathbb{F}_{0}$ is a strictly exponentially preinvex-FM on $K_{\partial}$, then $\Omega$ is a singleton set or empty. That is, if $\mathcal{T}$ is strictly exponentially preinvex-FM, then $\mathcal{T}$ has at least one global minimum. 
Proof. (1). Let $\mathcal{T}$ be exponentially preinvex-FM. If $\Omega$ is an empty set, then $\Omega$ is an invex set. Assume that, $u, \vartheta \in \Omega$, that is $u, \vartheta \in K_{\partial}$ and $e^{\mathcal{T}(u)}=\theta=e^{\mathcal{T}(\vartheta)}$. Since $\mathcal{T}$ is exponentially preinvex-FM,

$$
\begin{aligned}
e^{\mathcal{T}(u+\tau \partial(\vartheta, u))} & \preceq(1-\tau) e^{\mathcal{T}(u)} \widetilde{+} \tau e^{\mathcal{T}(\vartheta)}, \\
& =(1-\tau) \theta \widetilde{+} \tau \theta=\theta,
\end{aligned}
$$

for all $\tau \in(0,1)$. Hence, all points of the form $u+\tau \partial(\vartheta, u) \in \Omega, \tau \in[0,1]$, which implies that $\Omega$ is an invex set.

(2). Let $\mathcal{T}$ be a strictly exponentially preinvex-FM on $K_{\partial}$. Contrary suppose that there exist distinct points $u, \vartheta \in \Omega$ such that $e^{\mathcal{T}(u)}=\theta=e^{\mathcal{T}(\vartheta)}$. Since $\mathcal{T}$ is strictly exponentially preinvexFM then, for all $u, \vartheta \in K_{\partial}$ and $\tau \in(0,1)$, we have

$$
\begin{aligned}
e^{\mathcal{T}(u+\tau \partial(\vartheta, u))} & \prec(1-\tau) e^{\mathcal{T}(u)} \tilde{+} \tau e^{\mathcal{T}(\vartheta)}, \\
& =(1-\tau) \theta \widetilde{+} \tau \theta=\theta,
\end{aligned}
$$

which implies that $\theta \neq \inf _{u \in K_{\partial}}\left(e^{\mathcal{T}(u)}\right)$, so this contradict the fact. Hence, the result follows.

Theorem 3.3. Let $K_{\partial}$ be a nonempty set. Suppose that $\mathcal{T}: K_{\partial} \rightarrow \mathbb{F}_{0}$ be an exponentially preinvexFM on $K_{\partial}$, with $\theta=\inf _{u \in K_{\partial}}\left(e^{\mathcal{T}(u)}\right)$ exists in $[0,1]$, and that the set

$$
\Omega=\left\{u: u \in K_{\partial}, e^{\mathcal{T}(u)}=\theta\right\} \neq \emptyset .
$$

If $u$ is local minimum of $\mathcal{T}$, then it is also a global minimum of $\mathcal{T}$ on $K_{\partial}$.

Proof. Let $\mathcal{T}: K_{\partial} \rightarrow \mathbb{F}_{0}$ be an exponentially preinvex-FM and $u$ is local minimum of $\mathcal{T}$. If $u$ is not global minimum of $\mathcal{T}$, then $u \notin \Omega$. By hypothesis, $\Omega \neq \emptyset$. If $\vartheta \in \Omega$, then we must have $\mathcal{T}(\vartheta) \prec \mathcal{T}(u)$. Since $\mathcal{T}$ is exponentially preinvex-FM then, for $\tau \in(0,1)$, we have

$$
e^{\mathcal{T}(u+\tau \partial(\vartheta, u))} \preceq(1-\tau) e^{\mathcal{T}(u)} \widetilde{T} \tau e^{\mathcal{T}(\vartheta)} \prec e^{\mathcal{T}(u)}, \text { because } \mathcal{T}(\vartheta) \prec \mathcal{T}(u),
$$

for any small positive number $\tau$, and this contradiction proves the required result.

Theorem 3.4. Let $\mathcal{T}: K_{\partial} \rightarrow \mathbb{F}_{0}$ be an exponentially quasi-preinvex-FM on $K_{\partial}$. If $u \in K_{\partial}$ is a strict local minimum of $\mathcal{T}$, then it is also a strict global minimum of $\mathcal{T}$ on $K_{\partial}$.

Proof. Let $u \in K_{\partial}$ is a strict local minimum of $\mathcal{T}$. Then by definition of strict local minimum, there exist a $\delta>0$ such that $\mathcal{T}(u) \prec \mathcal{T}(\vartheta)$ for all $\vartheta \in K_{\partial} \cap B_{\delta}(u)$. Assume contrary, that is, for some $\vartheta \in K_{\partial}$, we have $\mathcal{T}(u) \geq \mathcal{T}(\vartheta)$. Since $\mathcal{T}$ is an exponentially quasi-preinvex-FM then, for $\tau \in$ $[0,1]$, we have

$$
e^{\mathcal{T}(u+\tau \partial(\vartheta, u))} \leq \max \left(e^{\mathcal{T}(u)}, e^{\mathcal{T}(\vartheta)}\right)=e^{\mathcal{T}(u)},
$$


since $\mathcal{T}(u) \geq \mathcal{T}(\vartheta)$, we have

$$
e^{\mathcal{T}(u+\tau \partial(\vartheta, u))} \preceq e^{\mathcal{T}(u)}
$$

This contradicts that $u \in K_{\partial}$ is a strict local minimum of $\mathcal{T}$, and hence the results follow.

Definition 3.2. A set $K_{\partial} \subseteq \mathbb{R}^{2}$ is said to an invex set w.r.t. $\partial$, if

$$
\left(u+\tau \partial(\vartheta, u),(1-\tau) \beta_{1} \widetilde{+} \tau \beta_{2}\right) \in K_{\partial},
$$

$\left(u, \beta_{1}\right),\left(\vartheta, \beta_{2}\right) \in K_{\partial}, u, \vartheta \in \mathbb{R}, \tau \in[0,1]$.

The epigraph of exponentially preinvex-FM $\mathcal{T}: K_{\partial} \rightarrow \mathbb{F}_{0}$ can be given as

$$
E(\mathcal{T})=\left\{(u, \beta): u \in K_{\partial}, \beta \in \mathbb{F}_{0}, e^{\mathcal{T}(u)} \preceq \beta\right\} .
$$

We now give a characterization of exponentially preinvex-FMs in terms of its epigraph $E(\mathcal{T})$.

Theorem 3.5. A FM $\mathcal{T}: K_{\partial} \rightarrow \mathbb{F}_{0}$ is an exponentially preinvex-FM w.r.t. $\partial$, if and only if, its epigraph is an invex set w.r.t. $\widetilde{\partial}$, where $\tilde{\partial}: E(\mathcal{T}) \times E(\mathcal{T}) \rightarrow \mathbb{R} \times \Omega$ with

$$
\tilde{\partial}\left(\left(\vartheta, \beta_{2}\right),\left(u, \beta_{1}\right)\right)=\left(\partial(\vartheta, u), \beta_{2} \widetilde{+}(-1) \beta_{1}\right),
$$

for $\left(u, \beta_{1}\right),\left(\vartheta, \beta_{2}\right) \in E(\mathcal{T})$.

Proof. Let $\left(u, \beta_{1}\right),\left(\vartheta, \beta_{2}\right) \in E(\mathcal{T})$. Then $e^{\mathcal{T}(u)} \preceq \beta_{1}$ and $e^{\mathcal{T}(\vartheta)} \preceq \beta_{2}$. Since FM $\mathcal{T}: K_{\partial} \rightarrow \mathbb{F}_{0}$ is exponentially preinvex-FM w.r.t. $\partial$, so for all $u, \vartheta \in K_{\partial}$ and $\tau \in[0,1]$, we have

$$
e^{\mathcal{T}(u+\tau \partial(\vartheta, u))} \preceq(1-\tau) e^{\mathcal{T}(u)} \widetilde{+} \tau e^{\mathcal{T}(\vartheta)} \preceq(1-\tau) \beta_{1} \widetilde{+} \tau \beta_{2},
$$

from which it follows that

$$
\left(u+\tau \partial(\vartheta, u),(1-\tau) \beta_{1} \widetilde{+} \tau \beta_{2}\right) \in E(\mathcal{T})
$$

which implies that

$$
\begin{aligned}
\left(u+\tau \partial(\vartheta, u),(1-\tau) \beta_{1} \widetilde{+} \tau \beta_{2}\right) & =\left(u, \beta_{1}\right)+\tau\left(\partial(\vartheta, u), \beta_{2} \widetilde{+}(-1) \beta_{1}\right), \\
& =\left(u, \beta_{1}\right)+\tau \tilde{\partial}\left(\left(\vartheta, \beta_{2}\right),\left(u, \beta_{1}\right)\right) \in E(\mathcal{T}) .
\end{aligned}
$$

Hence, $E(\mathcal{T})$ is an invex set w.r.t. $\tilde{\partial}: E(\mathcal{T}) \times E(\mathcal{T}) \rightarrow \mathbb{R} \times \Omega$, with $\tilde{\partial}\left(\left(\vartheta, \beta_{2}\right),\left(u, \beta_{1}\right)\right)=$ $\left(\partial(\vartheta, u), \beta_{2} \widetilde{+}(-1) \beta_{1}\right)$ for $\left(u, \beta_{1}\right),\left(\vartheta, \beta_{2}\right) \in E(\mathcal{T})$.

Conversely, $E(\mathcal{T})$ is an invex set w.r.t. $\tilde{\partial}: E(\mathcal{T}) \times E(\mathcal{T}) \rightarrow \mathbb{R} \times \Omega$, with $\tilde{\partial}\left(\left(\vartheta, \beta_{2}\right),\left(u, \beta_{1}\right)\right)=$ $\left(\partial(\vartheta, u), \beta_{2} \widetilde{+}(-1) \beta_{1}\right)$ for $\left(\vartheta, \beta_{2}\right),\left(u, \beta_{1}\right) \in E(\mathcal{T})$. Since $\left(u, e^{\mathcal{T}(u)}\right),\left(\vartheta, e^{\mathcal{T}(\vartheta)}\right) \in E(\mathcal{T})$, we have for $\tau \in[0,1]$

$$
\left(u, e^{\mathcal{T}(u)}\right)+\tau \tilde{\partial}\left(\left(\vartheta, e^{\mathcal{T}(\vartheta)}\right),\left(u, e^{\mathcal{T}(u)}\right)\right) \in E(\mathcal{T}),
$$

since $\tilde{\partial}\left(\left(\vartheta, \beta_{2}\right),\left(u, \beta_{1}\right)\right)=\left(\partial(\vartheta, u), \beta_{2} \widetilde{+}(-1) \beta_{1}\right)$, then

$$
\left(u, e^{\mathcal{T}(u)}\right)+\tau\left(\partial(\vartheta, u), e^{\mathcal{T}(\vartheta)} \widetilde{f}(-1) e^{\mathcal{T}(u)}\right) \in E(\mathcal{T}),
$$


which implies that

$$
\left(u+\tau \partial(\vartheta, u),(1-\tau) e^{\mathcal{T}(u)} \widetilde{+} \tau e^{\mathcal{T}(\vartheta)}\right) \in E(\mathcal{T}),
$$

so we have

$$
e^{\mathcal{T}(u+\tau \partial(\vartheta, u))} \preceq(1-\tau) e^{\mathcal{T}(u)} \widetilde{+} \tau e^{\mathcal{T}(\vartheta)} .
$$

Hence, $\mathcal{T}: K_{\partial} \rightarrow \mathbb{F}_{0}$ is an exponentially preinvex-FM w.r.t. $\partial$.

Theorem 3.6. Let $\left\{\mathcal{J}_{j}\right\}_{j \in I}: K_{\partial} \rightarrow \mathbb{F}_{0}$ be a family of exponentially preinvex-FMs and bounded above on an invex set $K_{\partial}$. Then the mapping $\mathcal{T}(u)=\sup _{j \in I} \mathcal{T}_{j}(u)$ is an exponentially preinvex-FM on $K_{\partial}$.

Proof. Since each $\mathcal{T}_{j}$ is an exponentially preinvex-FM on the invex set $K_{\partial}$, its epigraph $E(\mathcal{T})=$ $\left\{(u, \beta): u \in K_{\partial}, \beta \in \mathbb{F}_{0}, e^{\mathcal{T}_{j}(u)} \preceq \beta, j \in I\right\}$ is an invex set w.r.t. $\tilde{\partial}$, by Theorem 3.5. Then their intersection

$$
\bigcap_{j \in I} E(\mathcal{T})=\left\{(u, \beta): u \in K_{\partial}, \beta \in \mathbb{F}_{0}, e^{\mathcal{T}_{j}(u)} \preceq \beta, j \in I\right\},
$$

is also an invex set w.r.t. $\partial$. Hence, $\mathcal{T}(u)=\sup _{\boldsymbol{j} \in I} \mathcal{T}_{j}(u)$ is an exponentially preinvex-FM.

Definition 3.3. Let $\mathcal{T}: K_{\partial} \rightarrow \mathbb{F}_{0}$ be an FM then the level set of $\mathcal{T}$ is denoted by $\mathcal{T}_{\beta}$ and defined as,

$$
\mathcal{T}_{\beta}=\left\{u: u \in K_{\partial}, e^{\mathcal{T}(u)} \leq \beta, \beta \in \mathbb{F}_{0}\right\} .
$$

Note that the level set is also called $\beta$-cut of $\mathcal{T}$ and the set $\mathcal{T}_{\beta}$ generalizes the standard form of $\beta$ level of $\mathcal{T}$. The set of all level sets of $\mathcal{T}$ is represented as $L\left(\mathcal{T}_{\beta}\right)$.

Theorem 3.7. Let $\mathcal{T}: K_{\partial} \rightarrow \mathbb{F}_{0}$ be an exponentially preinvex-FM. Then $\mathcal{T}_{\beta}$ is an invex set.

Proof. Let $u, \vartheta \in \mathcal{T}_{\beta}$. Then $e^{\mathcal{T}(u)} \leq \beta$ and $e^{\mathcal{T}(\vartheta)} \leq \beta$. Since $\mathcal{T}: K_{\partial} \rightarrow \mathbb{F}_{0}$ is an exponentially preinvex-FM,

$$
e^{\mathcal{T}(u+\tau \partial(\vartheta, u))} \preceq(1-\tau) e^{\mathcal{T}(u)} \widetilde{+} \tau e^{\mathcal{T}(\vartheta)} \preceq(1-\tau) \beta+\tau \beta=\beta,
$$

hence $\mathcal{T}_{\beta}$ is an invex set.

Theorem 3.8. The comparable $\mathrm{FM} \mathcal{T}: K_{\partial} \rightarrow \mathbb{F}_{0}$ is said to be exponentially quasi-preinvex-FM if and only if, the level set $\mathcal{T}_{\beta}$ is an invex set.

Proof. Let $u, \vartheta \in \mathcal{T}_{\beta}$. Then by definition of $\mathcal{T}_{\beta}$, we have $e^{\mathcal{T}(u)} \leq \beta$ and $e^{\mathcal{T}(\vartheta)} \leq \beta$. Since $u, \vartheta \in K_{\partial}$ and $K_{\partial}$ is invex set then, $u+\tau \partial(\vartheta, u) \in K_{\partial}$. Since $\mathcal{T}$ is exponentially quasi-preinvex-FM then,

$$
e^{\mathcal{T}(u+\tau \partial(\vartheta, u))} \preceq \max \left(e^{\mathcal{T}(u)}, e^{\mathcal{T}(\vartheta)}\right) \preceq \max (\beta, \beta)=\beta, \forall u, \vartheta \in R, \tau \in[0,1],
$$

from which we can note that $u+\tau \partial(\vartheta, u) \in \mathcal{T}_{\beta}$ is an invex set. 
Conversely, assume that $\mathcal{T}_{\beta}$ be an invex set to prove $\mathcal{T}$ is exponentially quasi-preinvex-FM. Since $\mathcal{T}_{\beta}$ is an invex set then, for any $u, \vartheta \in \mathcal{T}_{\beta}$ such that $u+\tau \partial(\vartheta, u) \in \mathcal{T}_{\beta}$ with $\tau \in[0,1]$. Now we take $\max \left(e^{\mathcal{T}(u)}, e^{\mathcal{T}(\vartheta)}\right)=\beta$ and $e^{\mathcal{T}(u)} \geq e^{\mathcal{T}(\vartheta)}$.

Now by the definition of invex set $\mathcal{T}_{\beta}$, we have

$$
e^{\mathcal{T}(u+\tau \partial(\vartheta, u))} \leq \max \left(e^{\mathcal{T}(u)}, e^{\mathcal{T}(\vartheta)}\right) \leq \beta .
$$

Hence, $\mathcal{T}$ is exponentially quasi-preinvex-FM.

Lemma 3.1. For a comparable FM $\mathcal{T}: K_{\partial} \rightarrow \mathbb{F}_{0}$ :

Exponentially logarithmic preinvexity $\Rightarrow$ exponentially preinvexity $\Rightarrow$ exponentially quasipreinvexity.

Proof. For $\tau \in[0,1]$, and $u, \vartheta \in K_{\partial}$, we have

$$
\begin{aligned}
e^{\mathcal{T}(u+\tau \partial(\vartheta, u))} & \leq\left(e^{\mathcal{T}(u)}\right)^{1-\tau}\left(e^{\mathcal{T}(\vartheta)}\right)^{\tau} \\
& \leq(1-\tau) e^{\mathcal{T}(u)} \widetilde{T} \tau e^{\mathcal{T}(\vartheta)} \\
& \leq \max \left(e^{\mathcal{T}(u)}, e^{\mathcal{T}(\vartheta)}\right) .
\end{aligned}
$$

Theorem 3.9. Let FM $\mathcal{T}: K_{\partial} \rightarrow \mathbb{F}_{0}$ be a exponentially logarithmic preinvex-FM. Then its epigraph $E(\mathcal{T})$ is an invex set.

Proof. From Lemma 3.1, it follows that exponentially logarithmic preinvex-FM $\mathcal{T}$ is exponentially preinvex-FM. Hence by Theorem 3.5, epigraph $E(\mathcal{T})$ is an invex set.

Theorem 3.10. Let $\left\{\mathcal{T}_{j}\right\}_{j \in I}: K_{\partial} \rightarrow \mathbb{F}_{0}$ be a family of exponentially logarithmic preinvex-FMs and bounded above on an invex set $K_{\partial}$. Then the mapping $\mathcal{T}(u)=\sup _{j \in I} \mathcal{T}_{j}(u)$ is an exponentially logarithmic preinvex-FM on $K_{\partial}$.

Proof. By using Lemma 3.1 and Theorem 3.6, the demonstration of proof is analogous to Theorem 3.9.

\section{EXPONENTIALLY INVEX FUZZY MAPPINGS}

In this section, we propose the concept of exponentially-IFMs and some relations are established between exponentially preinvex-FM, exponentially-IFM and exponentially invex fuzzy monotone mapping.

Definition 4.1. A FM $\mathcal{T}: K_{\partial} \rightarrow \mathbb{F}(\mathbb{R})$ is called sharply exponentially pseudo invex w.r.t. $\partial$ on $K_{\partial}$, if 


$$
\left\langle e^{\mathcal{T}(u)} \mathcal{T}^{\prime}(u), \partial(\vartheta, u)\right\rangle \geq \tilde{0} \Longrightarrow e^{\mathcal{T}(\vartheta+\tau \partial(\vartheta, u))} \preceq e^{\mathcal{T}(\vartheta)},
$$

$\forall u, \vartheta \in K_{\partial}, \tau \in[0,1]$, where $\mathcal{T}$ ', is G-differentiable of $\mathcal{T}$ at $u$.

Theorem 4.1. Let $\mathcal{T}$ be a sharply exponentially pseudo preinvex-FM on $K_{\partial}$. Then

$$
\simeq\left\langle e^{\mathcal{T}(\vartheta)} \mathcal{T}^{\prime}(\vartheta), \partial(\vartheta, u)\right\rangle \geq \tilde{0}, \forall u, \vartheta \in K_{\partial} .
$$

Proof. Let $\mathcal{T}$ be a sharply exponentially pseudo preinvex-FM on $K_{\partial}$. Then for all $u, \vartheta \in K_{\partial}, \tau \in$ $[0,1]$, we have

$$
e^{\mathcal{T}(\vartheta)} \geq e^{\mathcal{T}(\vartheta+\tau \partial(\vartheta, u))},
$$

from which we get

$$
\frac{e^{\mathcal{T}(\vartheta+\tau \partial(\vartheta, u))} \simeq e^{\mathcal{T}(\vartheta)}}{\tau} \leq \tilde{0} .
$$

Taking limit in the above inequality as $\tau \rightarrow 0$, we obtain

$$
\simeq\left\langle e^{\mathcal{T}(\vartheta)} \mathcal{T}^{\prime}(\vartheta), \partial(\vartheta, u)\right\rangle \geq \tilde{0}, \forall u, \vartheta \in K_{\partial} .
$$

Hence, the result follows.

Definition 4.2. A G-differentiable FM $\mathcal{T}: K_{\partial} \rightarrow \mathbb{F}(\mathbb{R})$ is said to be:

- invex w.r.t. $\partial$, if

$$
e^{\mathcal{T}(\vartheta)} \simeq e^{\mathcal{T}(u)} \geq\left\langle e^{\mathcal{T}(u)} \mathcal{T} \cdot(u), \partial(\vartheta, u)\right\rangle,
$$

- $\quad$ strictly incave w.r.t. $\partial$, if inequality (4.1) is reversed.

- invex w.r.t. $\partial$, if

$$
e^{\mathcal{T}(\vartheta)} \simeq e^{\mathcal{T}(u)} \geq\left\langle e^{\mathcal{T}(u)} \mathcal{T} \cdot(u), \partial(\vartheta, u)\right\rangle,
$$

- $\quad$ strictly incave w.r.t. $\partial$, if inequality (4.2) is reversed.

$\forall u, \vartheta \in K_{\partial}$.

Example 4.1. We consider the FMs $\mathcal{T}:(0, \infty) \rightarrow \mathbb{F}(\mathbb{R})$ defined by,

$$
\mathcal{T}(u)(\sigma)=\left\{\begin{array}{cc}
\frac{4 \sigma-u}{u} & \sigma \in\left[\frac{1}{4} u, u\right], \\
\frac{3 u-2 \sigma}{u} & \sigma \in\left(u, \frac{3}{2} u\right], \\
0 & \text { otherwise. }
\end{array}\right.
$$

Then, for each $\gamma \in[0,1]$, we have $\mathcal{T}_{\gamma}(u)=\left[\left(\frac{1}{4}+\frac{3}{4} \gamma\right) u,\left(\frac{3}{2}-\frac{1}{2} \gamma\right) u\right]$. Since end point functions $\mathcal{T}_{*}(\gamma), \mathcal{T}^{*}(\gamma)$ are exponentially invex functions for each $\gamma \in[0,1]$, then $\mathcal{T}$ is exponentially-IFM w.r.t.

$$
\partial(\vartheta, u)=\vartheta
$$


Example 4.2. We consider the FMs $\mathcal{T}:(0, \infty) \rightarrow \mathbb{F}(\mathbb{R})$ defined by,

$$
\mathcal{T}(u)(\sigma)= \begin{cases}\frac{-u+\sigma}{u} & \sigma \in[u, 2 u], \\ \frac{4 u-\sigma}{2 u} & \sigma \in(2 u, 4 u], \\ 0 & \text { otherwise. }\end{cases}
$$

Then, for each $\gamma \in[0,1]$, we have $\mathcal{T}_{\gamma}(u)=[(1+\gamma) u, 2(2-\gamma) u]$. Since end point functions $\mathcal{T}_{*}(\gamma)$, $\mathcal{T}^{*}(\gamma)$ are exponentially invex functions for each $\gamma \in[0,1]$, then $\mathcal{T}$ is strictly exponentially-IFM w.r.t.

$$
\partial(\vartheta, u)=\vartheta+u
$$

Theorem 4.2. Let $\mathcal{T}: K_{\partial} \rightarrow \mathbb{F}(\mathbb{R})$ be a G-differentiable FM and let

$$
\partial(\vartheta, u+\tau \partial(\vartheta, u))=(1-\tau) \partial(\vartheta, u)
$$

$$
\partial(u, u+\tau \partial(\vartheta, u))=-\tau \partial(\vartheta, u) .
$$

Then $\mathcal{T}$ is an exponentially preinvex-FM, if and only if $\mathcal{T}$ is an exponentially-IFM.

Proof. Let $\mathcal{T}: K_{\partial} \rightarrow \mathbb{F}(\mathbb{R})$ be G-differentiable exponentially preinvex-FM. Since $\mathcal{T}$ is exponentially preinvex-FM then, for each $u, \vartheta \in K_{\partial}$ and $\tau \in[0,1]$, therefore

$$
\begin{aligned}
e^{\mathcal{T}(u+\tau \partial(\vartheta, u))} & \leq(1-\tau) e^{\mathcal{T}(u)} \widetilde{+} \tau e^{\mathcal{T}(\vartheta)}, \\
& =e^{\mathcal{T}(u)} \widetilde{+} \tau\left(e^{\mathcal{T}(\vartheta)} \simeq e^{\mathcal{T}(u)}\right),
\end{aligned}
$$

which implies that

$$
\begin{aligned}
& \tau\left(e^{\mathcal{T}(\vartheta)} \simeq e^{\mathcal{T}(u)}\right) \geq e^{\mathcal{T}(u+\tau \partial(\vartheta, u))} \simeq e^{\mathcal{T}(u)}, \\
& e^{\mathcal{T}(\vartheta)} \simeq e^{\mathcal{T}(u)} \geq \frac{e^{\mathcal{T}(u+\tau \partial(\vartheta, u))} \simeq e^{\mathcal{T}(u)}}{\tau} .
\end{aligned}
$$

On taking limit in the above inequality as $\tau \rightarrow 0$, we obtain

$$
e^{\mathcal{T}(\vartheta)} \simeq e^{\mathcal{T}(u)} \geq\left\langle e^{\mathcal{T}(u)} \mathcal{T}^{\prime}(u), \partial(\vartheta, u)\right\rangle .
$$

Conversely, let $\mathcal{T}$ be an exponentially-IFM. Since $K_{\partial}$ is an invex set so we have, $\vartheta_{\tau}=u+$ $\tau \partial(\vartheta, u) \in K_{\partial}$ for all $u, \vartheta \in K_{\partial}$ and $\tau \in[0,1]$. Taking $\vartheta=\vartheta_{\tau}$ in (4.1), we get

$$
e^{\mathcal{T}\left(\vartheta_{\tau}\right)} \simeq e^{\mathcal{T}(u)} \geq\left\langle e^{\mathcal{T}(u)} \mathcal{T}^{\prime}(u), \partial\left(\vartheta_{\tau}, u\right)\right\rangle .
$$

From (i) and (ii), we have

$$
e^{\mathcal{T}\left(\vartheta_{\tau}\right)} \simeq e^{\mathcal{T}(u)} \geq(1-\tau)\left\langle e^{\mathcal{T}(u)} \mathcal{T} \cdot(u), \partial(\vartheta, u)\right\rangle .
$$

In a similar way, we get

$$
\begin{aligned}
e^{\mathcal{T}(u) \simeq e^{\mathcal{T}\left(\vartheta_{\tau}\right)}} & \geq\left\langle e^{\mathcal{T}(u)} \mathcal{T}^{\prime}(u), \partial\left(u, \vartheta_{\tau}\right)\right\rangle \\
& =-\tau\left\langle e^{\mathcal{T}(u)} \mathcal{T}^{\prime}(u), \partial\left(\vartheta, \vartheta_{\tau}\right)\right\rangle,
\end{aligned}
$$

Multiplying (4.3) by $\tau$ and (4.4) by $(1-\tau)$, and adding the resultant, we have 


$$
e^{\mathcal{T}\left(\vartheta_{\tau}\right)} \preceq(1-\tau) e^{\mathcal{T}(u)} \widetilde{+} \tau e^{\mathcal{T}(\vartheta)},
$$

which implies that

$$
e^{\mathcal{T}(u+\tau \partial(\vartheta, u))} \preceq(1-\tau) e^{\mathcal{T}(u)} \widetilde{+} \tau e^{\mathcal{T}(\vartheta)} .
$$

Hence, $\mathcal{T}$ is exponentially preinvex-FM w.r.t. $\partial$.

Theorem 4.3. Let $\mathcal{T}$ be a G-differentiable exponentially preinvex-FM on the invex set $K_{\partial}$ and let

$$
\partial(\vartheta, u+\tau \partial(\vartheta, u))=(1-\tau) \partial(\vartheta, u), \quad \text { (ii) } \quad \partial(u, u+\tau \partial(\vartheta, u))=-\tau \partial(\vartheta, u) .
$$

Then the followings are equivalent

(1) $e^{\mathcal{T}(\vartheta)} \simeq e^{\mathcal{T}(u)} \geq\left\langle e^{\mathcal{T}(u)} \mathcal{T},(u), \partial(\vartheta, u)\right\rangle$,

(2) $\left\langle e^{\mathcal{T}(u)} \mathcal{T}^{\prime}(u), \partial(\vartheta, u)\right\rangle \widetilde{+}\left\langle e^{\mathcal{T}(\vartheta)} \mathcal{T}^{\prime}(\vartheta), \partial(u, \vartheta)\right\rangle \leq \tilde{0}$,

for all $u, \vartheta \in K_{\partial}$.

Proof. (1) implies (2), let (1) holds. Then, by replacing $\vartheta$ by $u$ and $u$ by $\vartheta$ in (4.5), we get

$$
e^{\mathcal{T}(u)} \simeq e^{\mathcal{T}(\vartheta)} \geq\left\langle e^{\mathcal{T}(\vartheta)} \mathcal{T}^{\prime}(\vartheta), \partial(u, \vartheta)\right\rangle,
$$

adding (4.5) and (4.7), we have

$$
\left\langle e^{\mathcal{T}(u)} \mathcal{T}^{\prime}(u), \partial(\vartheta, u)\right\rangle \widetilde{+}\left\langle e^{\mathcal{T}(\vartheta)} \mathcal{T}^{\prime}(\vartheta), \partial(u, \vartheta)\right\rangle \leq \tilde{0},
$$

(2) implies (1), assume that (2) holds. Then

$$
\left\langle e^{\mathcal{T}(u)} \mathcal{T}^{\prime}(u), \partial(\vartheta, u)\right\rangle \preceq \simeq\left\langle e^{\mathcal{T}(\vartheta)} \mathcal{T},(\vartheta), \partial(u, \vartheta)\right\rangle,
$$

Since $K_{\partial}$ is an invex set so we have, $\vartheta_{\tau}=u+\tau \partial(\vartheta, u) \in K_{\partial}$ for all $u, \vartheta \in K_{\partial}$ and $t \in[0,1]$. Taking $\vartheta=\vartheta_{\tau}$ in $(4.8)$, we obtain

$$
\left\langle e^{\mathcal{T}(u+\tau \partial(\vartheta, u))} \mathcal{T}^{\prime}(u+\tau \partial(\vartheta, u)), \partial(u, u+\tau \partial(\vartheta, u))\right\rangle \preceq \simeq\left\langle e^{\mathcal{T}(u)} \mathcal{T}^{\prime}(u), \partial(u+\tau \partial(\vartheta, u), u)\right\rangle,
$$

From (i) and (ii), we have

$$
\begin{gathered}
\left\langle e^{\mathcal{T}(u+\tau \partial(\vartheta, u))} \mathcal{T}^{\prime}(u+\tau \partial(\vartheta, u)), \tau \partial(\vartheta, u)\right\rangle \geq\left\langle e^{\mathcal{T}(u)} \mathcal{T}^{\prime}(u), \tau \partial(\vartheta, u)\right\rangle, \\
\left\langle e^{\mathcal{T}(u+\tau \partial(\vartheta, u))} \mathcal{T}^{\prime}(u+\tau \partial(\vartheta, u)), \partial(\vartheta, u)\right\rangle \geq\left\langle e^{\mathcal{T}(u)} \mathcal{T}^{\prime}(u), \partial(\vartheta, u)\right\rangle,
\end{gathered}
$$

Let

$$
\mathcal{H}(\tau)=e^{\mathcal{T}(u+\tau \partial(\vartheta, u))} .
$$

Taking G-derivative w.r.t. $\tau$, we get

$$
\mathcal{H}^{\prime}(\tau)=e^{\mathcal{T}(u+\tau \partial(\vartheta, u))} \mathcal{T}^{\prime}(u+\tau \partial(\vartheta, u)) \cdot \partial(\vartheta, u)=\left\langle e^{\mathcal{T}(u+\tau \partial(\vartheta, u))} \mathcal{T}^{\prime}(u+\tau \partial(\vartheta, u)), \partial(\vartheta, u)\right\rangle .
$$

The above inequality along with (4.9) gives

$$
\mathcal{H}^{\prime}(\tau) \geq\left\langle e^{\mathcal{T}(u)} \mathcal{T}^{\prime}(u), \partial(\vartheta, u)\right\rangle,
$$

By integrating (4.10) between 0 to 1 w.r.t. $\tau$, we get 


$$
\begin{gathered}
\mathcal{H}(1) \simeq \mathcal{H}(0) \geq\left\langle e^{\mathcal{T}(u)} \mathcal{T} \mathcal{T}^{\prime}(u), \partial(\vartheta, u)\right\rangle, \\
e^{\mathcal{T}(u+\partial(\vartheta, u))} \simeq e^{\mathcal{T}(u)} \geq\left\langle e^{\mathcal{T}(u)} \mathcal{T},(u), \partial(\vartheta, u)\right\rangle .
\end{gathered}
$$

Since $\mathcal{T}$ is strongly exponentially preinvex-FM, then for $\tau=1$, we have

$$
e^{\mathcal{T}(\vartheta)} \simeq e^{\mathcal{T}(u)} \geq\left\langle e^{\mathcal{T}(u)} \mathcal{T},(u), \partial(\vartheta, u)\right\rangle, \text { for all } u, \vartheta \in K_{\partial} .
$$

Note that, if $\partial(\vartheta, u)=\vartheta-u$ in (4.5), then we obtain the G-differentiable exponentially convex-

FM. Consequently, the exponentially-IFM is more general than G-differentiable convex-FM. The exponentially-IFM type is equivalent to the type of FM whose stationary points are global minima.

\section{APPLICATIONS}

In this section, by using the familiar fact that fuzzy variational inequality problem has deep relationship with the fuzzy optimization problems, we try to explore some applications of exponentially preinvex-FMs in fuzzy optimization and prove that the minimum of PFMs can be distinguish with variational-like inequality.

Theorem 5.1. Let $\mathcal{T}$ be an exponentially preinvex-FM on $K_{\partial}$. Then $u \in K_{\partial}$ is the minimum of the FM $\mathcal{T}$, if and only if, for $u \in K_{\partial}$ such that

$$
\left\langle e^{\mathcal{T}(u)} \mathcal{T},(u), \partial(\vartheta, u)\right\rangle \geq \tilde{0}
$$

for all $\vartheta \in K_{\partial}$.

Proof. Let $u \in K_{\partial}$ be a minimum of $\mathcal{T}$. Then

$$
\mathcal{T}(u) \preceq \mathcal{T}(\vartheta), \quad \forall \vartheta \in K_{\partial},
$$

From which, we have

$$
e^{\mathcal{T}(u)} \preceq e^{\mathcal{T}(\vartheta)}, \forall \vartheta \in K_{\partial} .
$$

Since $K_{\partial}$ is an invex set, for all $u, \vartheta \in K_{\partial}, \tau \in[0,1], \vartheta_{\tau}=u+\tau \partial(\vartheta, u) \in K_{\partial}$. Taking $\vartheta=\vartheta_{\tau}$ in (5.2), we obtain

$$
\tilde{0} \preceq \frac{e^{\mathcal{T}(u+\tau \partial(\vartheta, u))} \simeq e^{\mathcal{T}(u)}}{\tau},
$$

Taking limit in the above inequality as $\tau \rightarrow 0$, we get

$$
\tilde{0} \leq\left\langle e^{\mathcal{T}(u)} \mathcal{T} \cdot(u), \partial(\vartheta, u)\right\rangle .
$$

Conversely, since $\mathcal{T}: K_{\partial} \rightarrow \mathbb{F}(\mathbb{R})$ is an exponentially preinvex-FM, so

$$
e^{\mathcal{T}(u+\tau \partial(\vartheta, u))} \preceq(1-\tau) e^{\mathcal{T}(u)} \widetilde{\mp} \tau e^{\mathcal{T}(\vartheta)},
$$




$$
e^{\mathcal{T}(\vartheta)} \simeq e^{\mathcal{T}(u)} \geq \frac{e^{\mathcal{T}(u+\tau \partial(\vartheta, u))} \simeq e^{\mathcal{T}(u)}}{\tau}
$$

again taking limit in the above inequality as $\tau \rightarrow 0$, we get

$$
e^{\mathcal{T}(\vartheta)} \simeq e^{\mathcal{T}(u)} \geq\left\langle e^{\mathcal{T}(u)} \mathcal{T}^{\prime}(u), \partial(\vartheta, u)\right\rangle,
$$

The above inequality and (5.1) yield

$$
e^{\mathcal{T}(\vartheta)} \simeq e^{\mathcal{T}(u)} \geq \tilde{0} .
$$

Hence, the result follows.

Remark 5.1. The inequality of the type (5.1) is called exponentially fuzzy variational-like inequality. We emphasize that if $\partial(\vartheta, u)=\vartheta-u$, the optimality conditions of the exponentially convex-FM can be characterized by the following inequality

$$
\left\langle e^{\mathcal{T}(u)} \mathcal{T},(u), \vartheta-u\right\rangle \geq \tilde{0}, \forall u, \vartheta \in K_{\partial},
$$

which is called exponentially fuzzy variational inequality.

We consider the functional $I(\vartheta)$, defined as

$$
I(\vartheta)=\mathcal{T}(\vartheta) \widetilde{+} \mathcal{J}(\vartheta), \forall \vartheta \in \mathbb{R},
$$

where $\mathcal{T}$ is a G-differentiable exponentially preinvex-FM and $\mathcal{J}$ is a non G-differentiable exponentially preinvex-FM.

Since both $\mathcal{T}(\vartheta)$ and $\mathcal{J}(\vartheta)$ both are exponentially preinvex-FMs, then by Remark 2.2 (iii), (5.3) can be written as,

$$
I(\vartheta)=e^{\mathcal{T}(\vartheta)} \widetilde{\leftarrow} e^{\mathcal{J}(\vartheta)}, \forall \vartheta \in \mathbb{R},
$$

where $e^{\mathcal{T}(\vartheta)}$ and $e^{\mathcal{J}(\vartheta)}$ both are preinvex-FM w.r.t. same $\partial$.

We know that the minimum of the functional $I(\vartheta)$, can be characterized by a class of variational like-inequalities.

Theorem 5.2. Let $\mathcal{T}: K_{\partial} \rightarrow \mathbb{F}(\mathbb{R})$ be a G-differentiable exponentially preinvex-FM and $\mathcal{J}: K_{\partial} \rightarrow$ $\mathbb{F}(\mathbb{R})$ be a non G-differentiable exponentially preinvex-FM. Then the functional $I(\vartheta)$ has minimum $u \in K_{\partial}$, if and only if, $u \in K_{\partial}$ satisfies

$$
\left\langle e^{\mathcal{T}(u)} \mathcal{T}^{\prime}(u), \partial(\vartheta, u)\right\rangle \widetilde{+} e^{\mathcal{J}(\vartheta)} \simeq e^{\mathcal{J}(u)} \geq \tilde{0}, \forall \vartheta \in K_{\partial} .
$$

Proof. Let $u \in K_{\partial}$ be the minimum of $I$ then by definition, for all $\vartheta \in K_{\partial}$ we have

$$
I(u) \preceq I(\vartheta) .
$$

Since $K_{\partial}$ is an invex set so $\vartheta_{\tau}=u+\tau \partial(\vartheta, u)$, for all $u, \vartheta \in K_{\partial}$ and $\tau \in[0,1]$. Replacing $\vartheta$ by $\vartheta_{\tau}$ in (5.6), we get 


$$
I(u) \preceq I(u+\tau \partial(\vartheta, u)),
$$

which implies that, using (5.4), we have

$$
e^{\mathcal{J}(u)} \widetilde{+} e^{\mathcal{J}(u)} \leq e^{\mathcal{T}((u+\tau \partial(\vartheta, u))} \widetilde{\mp} e^{\mathcal{J}((u+\tau \partial(\vartheta, u))} .
$$

Since $\mathcal{J}$ is exponentially preinvex-FM then,

$$
e^{\mathcal{T}(u)} \widetilde{\Psi} e^{\mathcal{J}(u)} \preceq e^{\mathcal{T}((u+\tau \partial(\vartheta, u))} \widetilde{\Psi}(1-\tau) e^{\mathcal{J}(u)} \widetilde{\mp} \tau e^{\mathcal{J}(\vartheta)},
$$

that is

$$
\tilde{0} \preceq e^{\mathcal{T}((u+\tau \partial(\vartheta, u))} \simeq e^{\mathcal{T}(u)} \widetilde{+} \tau\left(e^{\mathcal{J}(\vartheta)} \simeq e^{\mathcal{J}(u)}\right) .
$$

On dividing by " $\tau$ " and taking $\lim _{\tau \rightarrow 0}$, we get

$$
\tilde{0} \preceq \lim _{\tau \rightarrow 0} \frac{e^{\mathcal{T}((u+\tau \partial(\vartheta, u))} \simeq e^{\mathcal{T}(u)}}{\tau} \widetilde{\mp} e^{\mathcal{J}(\vartheta)} \simeq e^{\mathcal{J}(u)} .
$$

Conversely, let (5.5) be satisfy to prove $u \in K_{\partial}$ is a minimum of $I$. Assume that

$$
\tilde{0} \preceq\left\langle e^{\mathcal{T}(u)} \mathcal{T} \cdot(u), \partial(\vartheta, u)\right\rangle \widetilde{+} e^{\mathcal{J}(\vartheta)} \simeq e^{\mathcal{J}(u)} .
$$

for all $\vartheta \in K_{\partial}$, we have

$$
\begin{aligned}
& I(u) \simeq I(\vartheta)=e^{\mathcal{T}(u)} \widetilde{\mp} e^{\mathcal{J}(u)} \simeq e^{\mathcal{T}(\vartheta)} \simeq e^{\mathcal{J}(\vartheta)}, \\
& =\simeq\left(e^{\mathcal{T}(\vartheta)} \simeq e^{\mathcal{T}(u)}\right) \widetilde{\Psi} e^{\mathcal{J}(u)} \simeq e^{\mathcal{J}(\vartheta)},
\end{aligned}
$$

Using Theorem 4.2, we obtain

$$
I(u) \simeq I(\vartheta) \preceq \simeq\left[\left\langle e^{\mathcal{T}(u)} \mathcal{T}^{\prime}(u), \partial(\vartheta, u)\right\rangle \simeq e^{\mathcal{J}(\vartheta)} \widetilde{F} e^{\mathcal{J}(u)}\right] \preceq \tilde{0},
$$

From (5.5), we have

$$
I(u) \simeq I(\vartheta) \preceq \tilde{0}
$$

hence, $I(u) \leq I(\vartheta)$.

Note that the (5.5) is called exponentially fuzzy mixed variational like-inequality. This result shows that the fuzzy optimality conditions of fuzzy functional $I(\vartheta)$ can be characterized by exponentially fuzzy mixed variational-like inequalities. If $\partial(\vartheta, u)=\vartheta-u$, then from (5.1) and (5.2), we can obtain the family of some new classes.

\section{CONCLUSION}

Convex and generalized convex-FMs play an important role in fuzzy optimization. Therefore, by the importance of nonconvex-FMs, we have introduced and considered a class of nonconvex-FMs is called exponentially preinvex-FMs. It is illustrated that classical convexity 
and nonconvexity are special cases of exponentially preinvex-FMs. We have also introduced the notions of quasi-preinvex and log-preinvex-FMs and investigated some properties. Some relations among the exponentially preinvex-FMs, exponentially invex-FMs, and exponentially monotonicities are derived under some mild conditions. We have proved that optimality conditions of G-differentiable exponentially preinvex-FMs and for the sum of G-differentiable preinvex-FMs and non G-differentiable exponentially preinvex-FMs can be characterized by exponentially fuzzy variational-like inequalities and exponentially fuzzy mixed variational-like inequalities, respectively. The inequalities (5.1) and (5.2) are the interesting outcome of our main results. It is itself an engaging problem to flourish some well-organized some numerical methods for solving exponentially fuzzy variational-like inequalities and exponentially fuzzy mixed variational-like inequalities together with applications in applied and pure sciences. In the future, we will try to investigate the applications of exponentially fuzzy variational-like inequalities and exponentially fuzzy mixed variational-like inequalities in existence theory. We hope that these concepts and applications will be helpful for other authors to pay their roles in different fields of sciences.

Conflicts of Interest: The authors declare that there are no conflicts of interest regarding the publication of this paper.

\section{REFERENCES}

[1] M. Adamek, On a problem connected with strongly convex functions, Math. Inequal. Appl. 19 (2016), 1287-1293.

[2] N. I. Akhiezer, The classical moment problem and some related questions in analysis, Oliver and Boyd, Edinburgh, 1965.

[3] G. Alirezaei, R. Mazhar, On exponentially concave functions and their impact in information theory, Inform. Theory Appl. Workshop, 9 (2018), 265-274.

[4] T. Antczak, (p,r)-Invex sets and functions, J. Optim. Theory Appl. 263 (2001), 355-379.

[5] M. Avriel, r-Convex functions, Math. Program. 2 (1972), 309-323.

[6] M. U. Awan, M. A. Noor, E. Set, et al. On strongly (p, h)-convex functions, TWMS J. Pure Appl. Math. 9 (2019), 145-153. 
[7] A. Azcar, J. Gimnez, K. Nikodem, J. L. Sánchez, On strongly midconvex functions, Opuscula Math. 31 (2011), 15-26.

[8] B. Bede, S. G. Gal, Generalizations of the differentiability of fuzzy-number-valued functions with applications to fuzzy differential equations, Fuzzy Sets Syst. 151(3) (2005), 581-599.

[9] A. Ben-Isreal, B. Mond, What is invexity? Anziam J. 28 (1986), 1-9.

[10] S. N. Bernstein, Sur les fonctions absolument monotones, Acta Math. 52 (1929), 1-66.

[11] S. S. L. Chang, L. A. Zadeh, On fuzzy mapping and control, IEEE Trans. Syst., Man, Cybern. SMC-2 (1972), 30-34.

[12] S. S. Chang, Variational Inequality and Complementarity Problems Theory and Applications. Shanghai Scientific and Technological Literature Publishing House, Shanghai, (1991).

[13] D. Dubois, H. Prade, Operations on fuzzy numbers. Int. J. Syst. Sci. 9 (1978), 613-626.

[14] N. Furukawa, Convexity and local Lipschitz continuity of fuzzy-valued mappings. Fuzzy Sets Syst. 93 (1998), 113-119.

[15] Jr, R. Goetschel, W. Voxman, Elementary fuzzy calculus. Fuzzy Sets Syst. 18 (1986), 31-43.

[16] M. A. Hanson, On sufficiency of the Kuhn-Tucker conditions, J. Math. Anal. Appl. 80 (1980), 545-550.

[17] M. V. Jovanovic, A note on strongly convex and strongly quasi convex functions, Math. Notes, 60 (1966), 584-585.

[18] S. Karamardian, The nonlinear complementarity problem with applications, Part 2. J. Optim. Theory Appl. 4 (1969), 167-181.

[19] J. Li, M. A. Noor, On characterizations of preinvex fuzzy mappings, Computers Math. Appl. 59 (2010), 933-940.

[20] M. S. Mohan, S. K. Neogy, On invex sets and preinvex functions, J. Math. Anal. Appl. 189 (1995), 901908.

[21] S. Nanda, K. Kar, Convex fuzzy mappings. Fuzzy Sets Syst. 48 (1992), 129-132.

[22] M. A. Noor, Fuzzy preinvex functions. Fuzzy Sets Syst. 64 (1994), 95-104.

[23] M. A. Noor, Variational-like inequalities, Optimization, 30 (1994), 323-330.

[24] M. A. Noor, Variational inequalities for fuzzy mappings. (III), Fuzzy Sets Syst. 110 (2000), 101-108.

[25] M. A. Noor, K. I. Noor, Exponentially convex functions, J. Orissa Math. Soc. 39 (2019), 33-51.

[26] M. A. Noor, K. I. Noor, Strongly exponentially convex functions, UPB Sci. Bull. Ser. A: Appl. Math. Phys. 81 (2019), 75-84.

[27] M. A. Noor, K. I. Noor, Strongly exponentially convex functions and their properties, J. Adv. Math. Stud. 12 (2019), 177-185. 
[28] M. A. Noor, K. I. Noor, Some properties of exponentially preinvex functions, FACTA Univ. (NIS) Ser. Math. Inform. 34 (2019), 941-955.

[29] A. Rufián-Lizana, Y. Chalco-Cano, R. Osuna-Gómez, G. Ruiz-Garzón, On invex fuzzy mappings and fuzzy variational-like inequalities, Fuzzy Sets Syst. 200 (2012), 84-98.

[30] A. Rufián-Lizana, Y. Chalco-Cano, G. Ruiz-Garzón, H. Román-Flores, On some characterizations of preinvex fuzzy mappings, Top, 22 (2014), 771-783.

[31] Y. R. Syau, On convex and concave fuzzy mappings. Fuzzy Sets Syst. 103 (1999), 163-168.

[32] L. Stefanini, B. Bede, Generalized Hukuhara differentiability of interval-valued functions and interval differential equations, Nonlinear Anal., Theory Meth. Appl. 71 (2009), 1311-1328.

[33] Z. Wu, J. Xu, Generalized convex fuzzy mappings and fuzzy variational-like inequality, Fuzzy Sets Syst. 160 (2009), 1590-1619.

[34] Y. X. Zhao, S. Y. Wang, L. Coladas Uria, Characterizations of r-convex functions, J. Optim. Theory Appl. 145 (2010), 186-195. 